Relations industrielles

Industrial Relations

\title{
Lois techniques et économiques de la production par R. Frisch, Dunod, Paris, 1963, 378 pages.
}

\section{Bertrand Belzile}

Volume 19, numéro 3, juillet 1964

URI : https://id.erudit.org/iderudit/1021281ar

DOI : https://doi.org/10.7202/1021281ar

Aller au sommaire du numéro

Éditeur(s)

Département des relations industrielles de l’Université Laval

ISSN

0034-379X (imprimé)

1703-8138 (numérique)

Découvrir la revue

Citer ce compte rendu

Belzile, B. (1964). Compte rendu de [Lois techniques et économiques de la production par R. Frisch, Dunod, Paris, 1963, 378 pages.] Relations industrielles / Industrial Relations, 19(3), 404-404. https://doi.org/10.7202/1021281ar

Tous droits réservés @ C Département des relations industrielles de l’Université Laval, 1964
Ce document est protégé par la loi sur le droit d'auteur. L'utilisation des services d'Érudit (y compris la reproduction) est assujettie à sa politique d'utilisation que vous pouvez consulter en ligne.

https://apropos.erudit.org/fr/usagers/politique-dutilisation/ 
Comme a écrit le T.R.P. Louis-M. Régis, O.P.: \& $C^{\prime}$ est dans le respect de cette hiérarchie des voleurs que notre pays se donnera simultanément la paix et la prospérité, au sein d'une unité politique jalousement fidèle ò préserver et à favoriser les deux tradi. tions culturelles qui constituent notre originalité ou milieu d'un monde voué à la monotonie de la standardisation.

\section{RECENSIONS - BOOK REVIEWS}

Lois techniques et économiques de la production par R. Frisch, Dunad, Paris, 1963, 378 poges.

Selon l'auteur, les lois de production représentent la façon dont le ou les produits répondent aux modifications quantitatives de leurs facteurs. De plus, on parle d'anolyse économique quand les prix du marché entrent en ligne de compte. Dans le présent ouvrage, l'idée dominante est celle d'optimum et le raisonnement procède de la logique fondamentale des lois de production.

Le développement des institutions sociales et politiques a fait des deux hypothèses que sont la libre concurrence ou le monopole, un dilemme formel dont les termes répondent de moins en moins à la réalité des faits. Afin de rendre davantage compte de la réalité, l'auteur procède à une classification des principaux types de stratégie que révèlent l'observation. II distingue ainsi deux grandes catégories qu'il intitule stratégies de fixation et stratégies de négociation. Pormi les principaux types de stratégies de fixation, l'auteur considère l'adaptation (autonome) par la quantité, l'adaptation stochastique par le prix, l'adaptation par le prix ou par la quantité soumise à l'élasticité, le formulateur d'option et enfin, I'acquéreur d'option. De plus, il onalyse distinctement les principaux objectifs suivants: minimisation des coûts, maximisation de la production, maximisation du profit et enfin, optimalisation du profit.

Dans une première partie, le professeur Frisch traite de divers concepts: qu'est-ce que la production? facteurs de production; technique de production et différents types de théorie de la production. Dans les trois autres parties, il anolyse trois situations différentes: production instantanée simple ne comportant que des facteurs continus, production instontanée simple avec facteurs limitatifs et production multiple. Dans chacune de ces parties, l'auteur considère ò tour de rôle l'aspect technique et l'aspect économique. Dans la section technique, il utilise des concepts bien connus tels que productivités marginales, accélérations de production, productivités moyennes; élasticités moyennes et, entre autres, un concept relativement nouveau, soit le coefficient de conversion. La dernière partie expose les éléments d'une théorie dynamique de la production.

Le présent ouvrage représente probablement une contribution originale à la théorie économique de l'entreprise. II faut, de plus, souligner le caractère très scientifique de ce livre de même que la clarté de l'exposé en général. L'expression en termes mathématiques pour une large part en rend cependant la lecture quelque peu difficile pour les non-initiés aux mathématiques.

Bertrand Belzile

Comment administrer une entreprise. Publication du Ministère du Commerce, Ottawa (Canada), 1963. 120 pp.

Ce petit fascicule n'est pas un traité d'administration des grandes entreprises, mais un recueil de conseils protiques adressés à ceux qui exploitent une petite entreprise ou songent à en fonder une. Ce recueil traite d'abord de l'importance de la petite entreprise dans l'économie et les responsabilités qui incombent au propriétaire. Au deuxième chapitre, on distingue et définit les formes légales que peuvent prendre les entreprises.

Le troisième chopitre est un relevé des facteurs dont il faut tenir compte au cours du lancement d'un atelier ou d'une usine et d'un commerce de détail, en particulier. L'accès aux matières premières, la maind'oeurre, les conditions de vie dans la localité, les moyens de transport, les zones de marchés, les impôts locaux, le choix d'une 